\title{
Effects of long range electronic interactions on a one-dimensional electron system
}

\author{
Sylvain Capponi and Didier Poilblanc \\ Laboratoire de Physique Quantique 8 Unité Mixte de Recherche 5626, C.N.R.S., \\ Université Paul Sabatier, 31062 Toulouse, France \\ Thierry Giamarchi \\ Laboratoire de Physique des Solides 6 Unité Mixte de Recherche 2, C.N.R.S., \\ Université Paris-Sud, 91405 Orsay Cedex, France
}

\begin{abstract}
The effects of a long range electronic potential on a one dimensional chain of spinless fermions are investigated by numerical techniques (Exact Diagonalisation of rings with up to 30 sites complemented by finite size analysis) and analytic calculations. Due to a competition between the $2 k_{F}$ oscillations in the density and the (very slow) log divergence of the long wavelength part of the Coulomb potential, the metallic character of the system is enhanced at intermediate (and up to quite large) lengthscales. Despite some similarities, we found that this quasi-metallic regime is not of the Luttinger Liquid type, as evidenced by strong deviations from predictions of conformal field theory and in agreement with the picture of a (very weakly pinned) Wigner crystal. When the strength of the Coulomb interaction is substancially increased, we observe a smooth cross-over to a strongly localized charge density wave.
\end{abstract}

PACS numbers: 71.10.Fd, 71.27.+a, 71.30.+h

\section{INTRODUCTION}

In one dimensional systems long range (LR) Coulomb potential plays a particularly important role in electronic models [1]. Indeed, as the dimensionality of an electronic system is reduced, charge screening become less effective in reducing the range of the electron-electron interactions. The importance of LR interactions between carriers has been highlighted in some experiments on $\mathrm{GaAs}$ quantum wires [2] or quasi-one-dimensional organic conductors 3, 4. The role of a $1 / r$ Coulomb repulsion on the long distance properties of electrons confined to a chain has been theoretically investigated using bosonisation techniques [5]. It was found that the charge correlations decay very slowly with distance suggesting that the ground state (GS) is similar to a classical Wigner crystal (WC) [6.5. In this analysis quantum fluctuations only lead to small oscillations of the particles away from the configuration of minimal potential energy. However, this previous study was made in the continuum limit and the role of the lattice in this context is unclear. Theoretical, both analytic [7] 10] or numerical [11], and experimental [12] results suggest that umklapp processes have a crucial importance in 1D for the Mott transition. In addition, the $q \rightarrow 0$ singular forward scattering interaction and the $2 k_{F}$ backward scattering might lead to competing effects. Although it is believed that, strictly speaking, for a longrange $1 / r$ potential of arbitrary magnitude, the lattice would always produce a pinning of the WC, such an effect is (a) only achieved for very large systems (for not too large interaction) due to its logarithmic nature and (b) is very weak. Hence, easy de-pinning of the WC can occur for mesoscopic systems at small but finite temperature.

In order to investigate the interplay between Coulomb interaction and umklapp scattering as well as their size dependent effects, thermodynamic observables are calculated on chains with a variety of electron-electron interactions using numerical techniques and compared to some analytic predictions based on the bosonisation method. As the range of the electronic potential increases, our computational analysis suggests that, at intermediate length scales, the metallic character of the system is enhanced. However, strong umklapp occurs at large Coulomb potential leading to an insulating Charge Density Wave (CDW) state. Based on our numerical data and on analytic calculations we argue in favor of a smooth cross-over between a weakly pinned WC (exhibiting metallic character at length scales and energy scales we are dealing with here) and a CDW insulator. We believe that the physical behaviors observed here for systems of sizes $L \sim 20-30$ are of particular relevance for sub-nanometric quantum wires or carbon nanotubes.

This article is organised as follows: In Sec. II we investigate the role of an extended (but finite) range interaction. Spectacular oscillations in e.g. the charge stiffness are observed numerically as the range of the interaction is increased. In Sec. III, properties of a full $1 / r$ long range potential are analysed. First, the finite size behaviors of the single particle and two particle charge gaps as well as the charge stiffness are studied. Behaviors reminiscent of a Luttinger Liquid (with charge gaps $\propto 1 / L$ and charge stiffness $\propto 1 / L^{2}$ [13]) are seen at small and intermediate interactions. Qualitative changes occur at very large 
Coulomb repulsion with clear signatures of the opening of a charge gap and a drastic reduction of the charge stiffness. Based on a conformal field theory analysis, we then analyse the role of umklapp scattering by calculating the (size dependent) anomalous exponent. In particular, we show that, even at small Coulomb potential, strong deviations from the Luttinger Liquid (LL) predictions occur. Lastly, the momentum dependent low energy spectrum as well as the single particle spectral function are computed confirming the cross-over scenario.

The model analyzed here consists of a single periodic chain of spinless fermions with $L$-sites and an extended interaction,

$$
\begin{aligned}
H & =-t \sum_{i}\left(c_{i}^{\dagger} c_{i+1}+h . c .\right) \\
& +\sum_{i>j} V_{i-j}\left(n_{i}-\bar{n}\right)\left(n_{j}-\bar{n}\right)
\end{aligned}
$$

where $n_{i}(\bar{n})$ is the local (average) electron density, and the rest of the notation is standard $(t=1$ is the unit of energy). Two types of potentials will be considered: (i) a plain LR Coulomb interaction $V_{i-j}=V /|i-j|$; (ii) an extended range potential, typically $V_{i-j}=V /|i-j|$ for $|i-j| \leq r_{\max }$ and $V_{i-j}=0$ otherwise, extending up to a distance $r_{\max }$. Using Exact Diagonalization techniques, 1D closed chains are here analyzed. These numerical results are compared to analytic predictions based on the bosonisation technique. A uniform ionic background has been included in Eq. (11) such that the electrostatic energy per unit volume remains finite. In the rest of the paper, electron densities $\bar{n}=\frac{1}{2}$ and $\bar{n}=\frac{1}{3}$ will be considered. Finite size scaling analysis are performed by considering rings of typical sizes $L=12,16,20,24$ and 28 [resp. $L=12,18,24$, and 30$]$ for $\bar{n}=\frac{1}{2}$ [resp. $\bar{n}=\frac{1}{3}$ ]. For an even number $N_{e}$ of particles closed shell configurations are used by taking antiperiodic boundary conditions $(\mathrm{ABC})$.

\section{FINITE RANGE POTENTIALS}

We start our analysis by first gradually varying the range of the electron interaction $V_{i-j}$. For a (not too large) finite range interaction, the system is expected to belong to the LL universallity class characterized by a linear collective charge mode (see Appendix). The corresponding charge velocity can easily be obtained from the slope of the low energy mode i.e. the energy difference between the first excited state at the smallest finite momentum $q=\frac{2 \pi}{L}$ and the GS. Numerically, it is found that the expected $1 / L^{2}$ behavior of the finite size corrections [13] is very well fulfilled so that a simple fit provides accurate extrapolated results shown in Fig. 1 as a function of the potential strength $V$. It is clear from this picture that the leading correction in $V$ is linear and that the successive components $V_{2 p}$ and $V_{2 p+1}$ of the potential have quite different effects on the increase of $u$ compared to the non-interacting case ( $u=v_{F}=2$ at half filling). Indeed, adding even distance interactions barely affect the slopes of $u$ vs $V$.

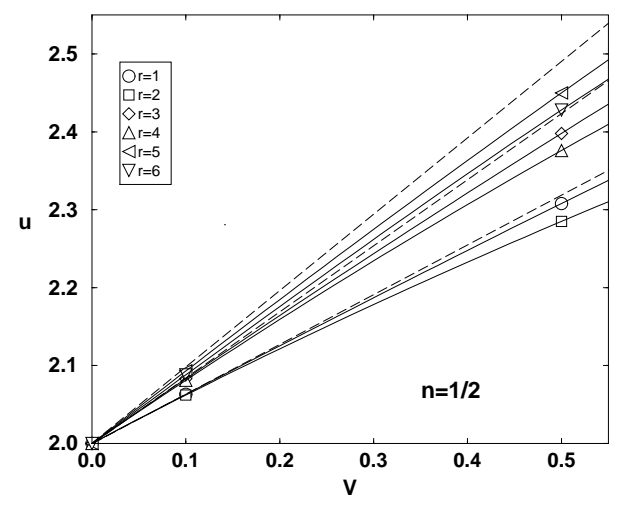

FIG. 1. Extrapolated velocity at density $\bar{n}=1 / 2$ vs $V$ for different ranges $r_{\max } \equiv r$ of the electron-electron repulsion. The dotted lines correspond to the theoretical predictions (3) for $r_{\max }=1$ or 2,3 or 4 , and 5 or 6 .

Some hint for this behavior can be found by using the continuum representation explained in Appendix A. As shown in the Appendix, because of the presence of a $2 k_{F}$ oscillations in the density, the various range components of the interaction interfere destructively. The bare Luttinger liquid parameters, describing the strength of the interactions are affected as (see (A6))

$$
\begin{aligned}
u / K & =v_{F}+\frac{2}{\pi} \sum_{r} V_{r}\left(1-\cos \left(2 k_{F} r\right)\right) \\
u K & =v_{F}
\end{aligned}
$$

For an half filled band $2 k_{F}=\pi$ and $v_{F}=2$. In (2) one can thus distinguish two contributions: (i) the one coming from the long wavelength part of the density $(q \sim 0)$ for which all interactions simply add. This is the term that is responsible for divergence associated with the long range Coulomb interaction since $\sum_{r} V_{r}$ diverges logarithmically in this case; (ii) the contribution coming from the $2 k_{F}$ part of the density. Because the density oscillates, interactions of different range can now interfere destructively. For long range interactions and large system sizes this term is bounded (it corresponds to the Fourier transform of the interaction potential with a Fourier component of $2 k_{F}$ ) and the system is thus totally dominated by the long wavelength part. For finite size systems and/or finite range interactions the correction coming from the $2 k_{F}$ term can be sizeable. In particular, at half filling one would get

$$
\begin{aligned}
u & \simeq 2+\frac{1}{\pi}\left(2 V_{1}+2 V_{3}+\cdots\right) \\
K & \simeq 1-\frac{1}{2 \pi}\left(2 V_{1}+2 V_{3}+\cdots\right)
\end{aligned}
$$


thus all even terms diseappear in $K$ and $u$ giving a $K[u]$ decreasing [increasing] less rapidly than if only the $q \sim 0$ part was concerned. In particular one would have $2 \mathrm{~V}$ in (3) for a nearest neighbor interaction and only $\sim 4.36 \mathrm{~V}$ for a 22 sites interaction.

The charge stiffness $D$ can be obtained numerically by threading a flux $\Phi$ (in unit of the flux quantum) through the ring. The curvature around the minimum of the GS energy $E_{0}(\Phi)$ provides the best estimate, namely $\left.D=\frac{1}{2} \partial^{2}\left(E_{0} / L\right) / \partial \phi^{2}\right)$ [where $\phi$ is a pseudo momentum $\phi=(2 \pi / L) \Phi]$. Note that, for an even number of electrons, the minimum occurs at $\Phi=1 / 2$ i.e. for antiperiodic boundary conditions corresponding to a noninteracting closed shell configuration. Results obtained on $L=16,20,24$ and 28-site rings with $N_{e}=L / 2$ electrons are shown in Fig. 2. We observe that the charge stiffness for $r_{\max }>1$ is systematically enhanced compared to the case of nearest neighbor (NN) repulsion $\left(r_{\max }=1\right)$. Note that for $r_{\max }=1$ a metal-insulator transition is known exactly to occur at $V=2$ so that $D$ in Fig. 2(a) [resp. Fig. 2(b)] extrapolates to a finite [resp. vanishing] value in that case. An interesting oscillatory behavior appears as $r_{\max }$ increases. For $r_{\max }=2 p+1$ (odd), $D$ monotonically increases with $p$. The maximum of the charge stiffness is obtained for $r_{\max }=2$ and $D$ decreases slightly for larger $r_{\max }=2 p^{\prime}$ (even). The (unique) limit corresponding to a long range $1 / r$ repulsion would then be reached from below (above) when $p \rightarrow \infty\left(p^{\prime} \rightarrow \infty\right)$. We believe that this feature is generic and is independent of the magnitude of $V$ as long as the potential is short range i.e. $r_{\max } \ll L$.

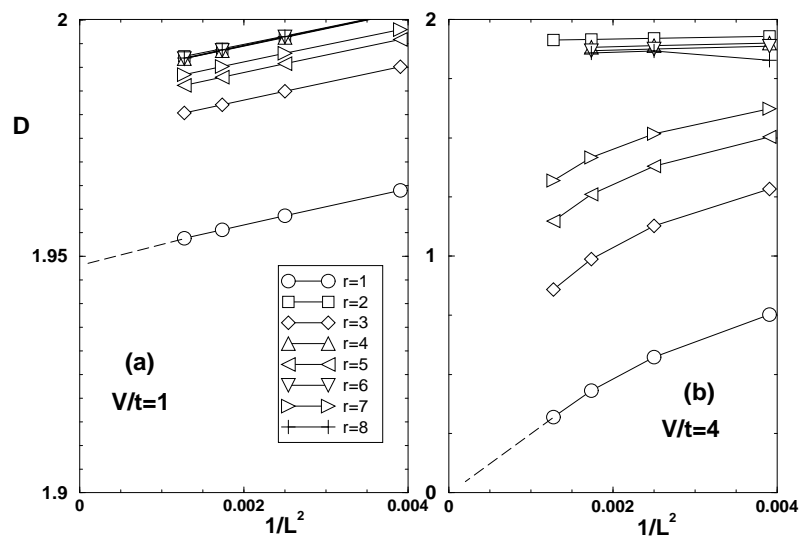

FIG. 2. Charge stiffness at density $\bar{n}=1 / 2$ vs inverse square length for different ranges $r_{\max } \equiv r$ of the electron-electron repulsion with amplitudes (a) $V=1$ and (b) $V=4$.

Our numerical results for $D$ can also be explained qualitatively using the bosonisation picture. Indeed, when more components $V_{p}$ of the potential are added, in addition to the change of the LL parameter $K$ described by (A6), a dramatic effect occurs also in the strength of the umklapp term (see Appendix)

$$
g=\sum_{r} V_{r} \cos \left(2 k_{F} r\right)
$$

At half filling, this term is reduced for all even range contributions (since one has $V_{1}-V_{2}, V_{1}-V_{2}+V_{3}-V_{4}$, etc. thus enhancing the metallic character of the system for intermediate sizes) [14]. Since without such umklapp term the stiffness $D$ is not renormalized by interactions $D=u K=v_{F}$ (see (2)), the strength of the umklapp is a direct measure of the reduction of the stiffness due to interaction effects (the stronger the umklapp term, the smaller the stiffness). For a second neighbor interaction the umklapp strength $g$ is at its minimum $V / 2$, consistent with the numerical findings of the largest stiffness. Note also that for second neighbor interactions $K$ is not decreased compared to the nearest neighbor one, thus allowing for a maximum enhancement of the charge stiffness. For odd range interactions the serie $V_{1}, V_{1}-V_{2}+V_{3}$, $V_{1}-V_{2}+V_{3}-V_{4}+V_{5}$ and so on is monotonically decreasing, whereas for even ranges $V_{1}-V_{2}, V_{1}-V_{2}+V_{3}-V_{4}$ and so on is increasing corresponding respectively to increasing and decreasing charge stiffness in good agreement with the numerical results. When the whole serie is included (for the Coulomb case) the umklapp strength is $g \sim 0.69 \mathrm{~V}$ and thus much weaker than the nearest neighbor one $g=V$.

Of course for larger length scales (2) is dominated by the logarithmic divergence coming from the $q \sim 0$ component whereas the $2 k_{F}$ part gives a finite contribution and $K \rightarrow 0$. Because of the presence of the umklapp term this always lead to an insulating gapped phase $\left(K<K_{c}\right)$. However both for intermediate sizes or finite range interactions the presence of long range interactions is in fact favorable to the metallic behavior.

\section{COULOMB 1/r POTENTIAL}

\section{A. Charge gaps}

We now turn to the case of the full $1 / r$ potential and to the investigation of the metal-insulator transition. The single particle charge gap at average density $\bar{n}=\frac{N_{e}}{L}$ is defined by $\Delta_{C, 1}=E_{0}\left(N_{e}+1\right)+E_{0}\left(N_{e}-1\right)-2 E_{0}\left(N_{e}\right)$. Note that the GS energies $E_{0}\left(N_{e} \pm 1\right)$ are calculated by keeping the ionic charge density constant (i.e. $\bar{n}$ fixed in Hamiltonian (11)). Our results for $\Delta_{C, 1}$ at $\bar{n}=1 / 2$ [resp. $\bar{n}=1 / 3$ ] are shown on Fig. 3 [resp. Fig. 价.

At small and intermediate interactions, the charge gap seems to vanish as $\sim 1 / L$, a behavior characteristic of a Luttinger Liquid (LL). However, we cannot exclude a very small extrapolated value. This suggests that the GS has a metallic character, at least approximately (i.e. with a very small charge gap). On the other hand, at large Coulomb repulsion, a charge gap clearly opens up. Note that at lower electron commensurability than half filling, a significantly larger interaction is needed to clearly see 


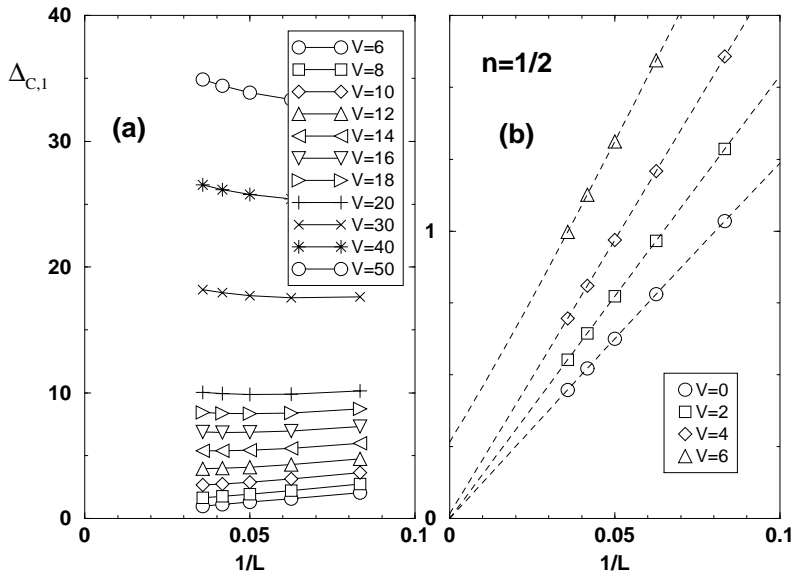

FIG. 3. Single particle charge gap vs $1 / L$ for $\bar{n}=1 / 2$ and several values of the LR Coulomb interaction $V$. Dotted lines are guides to the eye.

a signature of the gap: $V \sim 20$ at $\bar{n}=1 / 3$ compared to only $V \sim 5$ at $\bar{n}=1 / 2$.

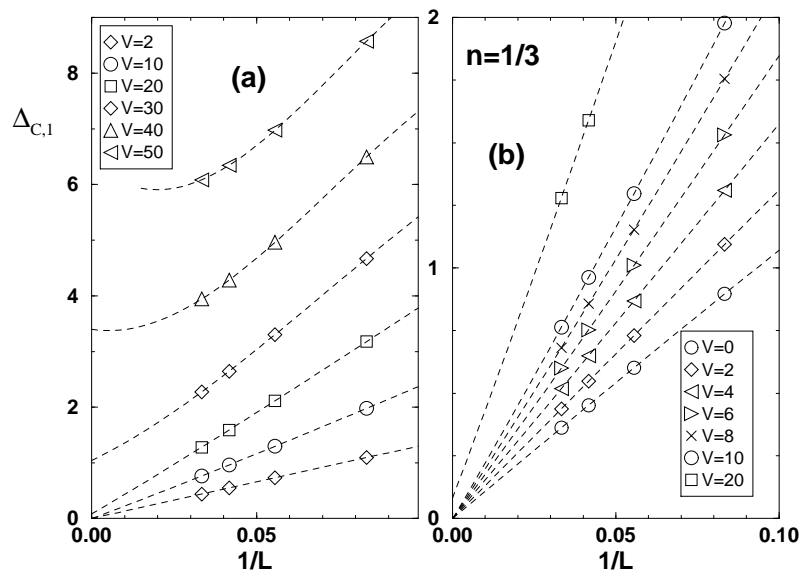

FIG. 4. Single particle charge gap vs $1 / L$ for $\bar{n}=1 / 3$ and several values of the LR Coulomb interaction $V$. Dotted lines are guides to the eye.

In order to estimate the sensitivity of the results to shell effects, we have also computed the two-particle charge gap at $\bar{n}=1 / 2$ defined by $\Delta_{C, 2}=E_{0}\left(N_{e}+2\right)+$ $E_{0}\left(N_{e}-2\right)-2 E_{0}\left(N_{e}\right)$ which only involves even number $N_{e}$ of particles. Results are shown in Fig. 5. For this range of parameters and system sizes, we observe, as expected, that $\Delta_{C, 2}$ approximatively equals $2 \Delta_{C, 1}$ although the additional finite size correction (typically $\sim 1 / L^{2}$ ) on top of the leading $1 / L$ term have a different sign.

Extrapolations of our data for $\Delta_{C, 1}$ to the thermodynamic limit have been attempted using a cubic spline (including higher powers of $1 / L$ if necessary) and the results are shown in Fig. 6. In the classical limit i.e. $t=0$, electron (hole) excitations are multiple massive fractional

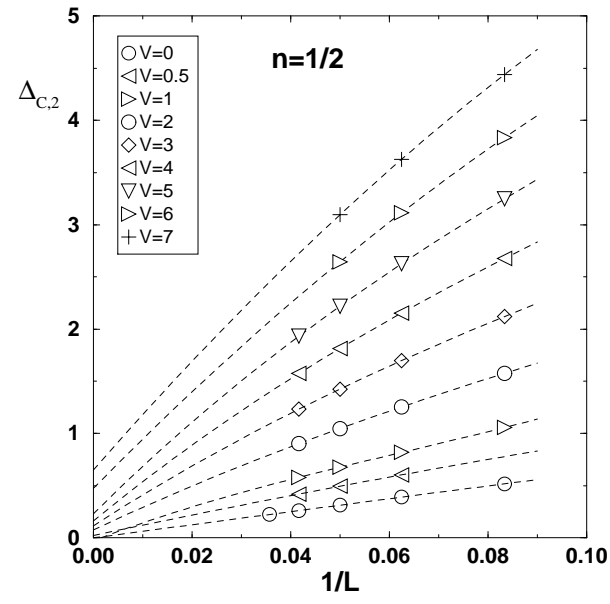

FIG. 5. Two-particle charge gap vs $1 / L$ for $\bar{n}=1 / 2$ and several values of the LR Coulomb interaction $V$. Dotted lines are guides to the eye.

charge excitations (solitons). For example, at half filling a dephasing of $\pi$ in the 01010101 etc. CDW pattern like 01011010 etc. produces a charge- $1 / 2$ excitation. An extra electron introduced in the system will then split into two such elementary excitations. Our numerical results at large $V$ agree well with the classical estimates e.g. $\Delta_{C, 1}=V$ at $\bar{n}=1 / 2$ or $\Delta_{C, 1}=\left(1-\frac{\pi \sqrt{3}}{9}\right) V$ at $\bar{n}=1 / 3$ [16]. We observe that a sizeable gap opens up in the LR model only at values of $V$ significantly larger than the critical values corresponding to the metal-insulator transitions of the related "screened" models where the range of the interaction is limited to $r_{\max }=\frac{1}{\bar{n}}-1\left(\frac{1}{\bar{n}}\right.$ being the average inter-particle distance). For example, at $\bar{n}=1 / 2$, the single particle gap for a LR potential of magnitude $V(V>4)$ is roughly of the same order than the gap produced by a short range NN interaction of magnitude $V / 2$. This clearly shows again that the effect of umklapp scattering is reduced in the presence of a LR interaction. However, we believe that, in the case of a LR $1 / r$ potential, a cross-over rather than a real transition occurs for increasing $V$.

\section{B. Charge stiffness}

Results for the charge stiffness of the $1 / r$ model at density $n=1 / 2$ are shown in Fig. 7. Note that we have used here two sets of closed rings of sizes $L=4 p(12$ to 28 sites) and $L=4 p+2$ (14 to 30 sites). $\mathrm{ABC}$ and $\mathrm{PBC}$ are used respectively to ensure $(V=0)$ closed shell configurations in all cases. However, we observe that the two sets of data exhibit slightly different scaling behaviors which can be attributed to small shell effects. In agreement with the previous results for the single particle gap, these data suggest an effective metallic behavior at small and intermediate magnitude $V$ while for fairly 


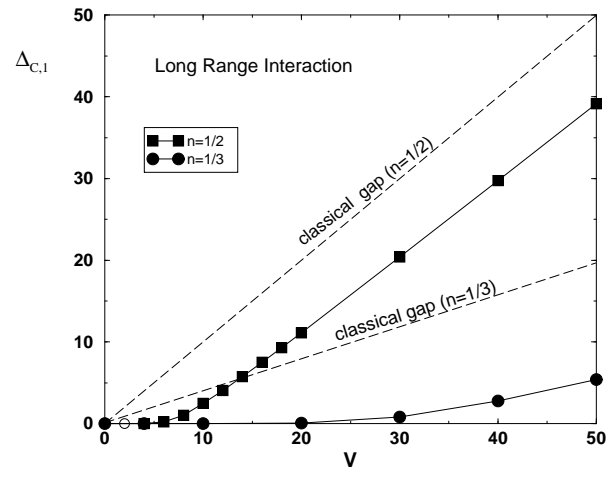

FIG. 6. Single particle charge gap for $\bar{n}=1 / 2$ and $\bar{n}=1 / 3$ as a function of the magnitude of the LR Coulomb interaction $V$. The dotted lines correspond to the classical behaviors $\Delta_{C, 1} \propto V$ valid in the $t=0$ limit.

large $V$ the charge stiffness is suppressed, even at these length scales. Note that, for, let us say, $V \leq 4$ the charge stiffness is particularly large, typically of the same order as in a non-interacting system. Since $D$ is directly proportional to the Drude weight in the optical conductivity, this signals that most of the optical weight lies at $\omega \sim 0$ (eventhough a tiny pinning frequency might exist). This contrasts with the large transfer of weight to finite frequencies occuring in the insulating phase of the $\mathrm{NN}$ t- $\mathrm{V}$ model for $V>2$.

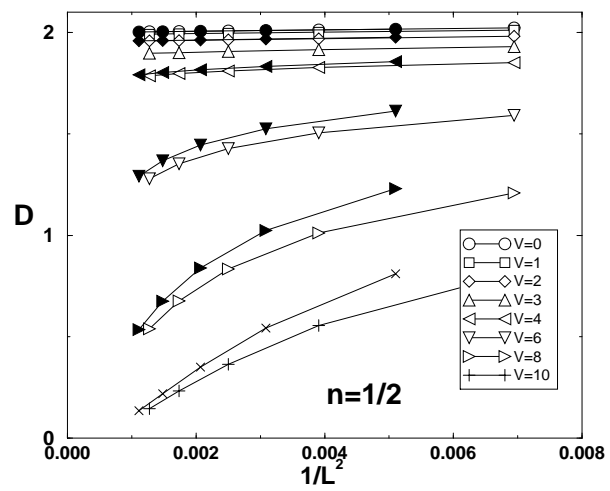

FIG. 7. Charge stiffness at density $\bar{n}=1 / 2$ vs inverse square length for a $1 / r$ long range potential of several magnitudes (as indicated on the picture). Open (closed) symbols correspond to an even (odd) number of electrons.

\section{Anomalous exponents}

Although the singular $q \rightarrow 0$ part of the $1 / r$ potential does not alone lead to true localisation, it is nevertheless responsible to a $K \rightarrow 0 \mathrm{LL}$ constant so that umklapp scattering becomes relevant and can lead to an insulating behavior. However, from the previous analysis, this effect is quite small at intermediate coupling $V$ and for the system sizes we considered here. Hence, we shall attempt here a more sensitive analysis based on identities from conformal field theories. Note that the latter are $a$ priori valid only in the case of standard Luttinger liquids (i.e. 1D models with short range interactions). Therefore, deviations from these expected relations will signal non LL behaviors.

To perform this analysis the charge velocity is needed. Results are shown in Fig. 8. The charge velocity increases with $V$ and system size as expected. In the continuum limit, the WC picture predicts a low energy dispersion of the form $q \log ^{1 / 2}(q)$. This logarithmic divergence comes directly from the $q \sim 0$ contribution of the potential to $u / K$ as shown in (2) since $\sum_{r} V_{r}$ is logarithmically divergent. We thus expect a very weak $\log ^{1 / 2}(1 / L)$ divergence of $u$ consistent with our data at sufficiently large $V$.

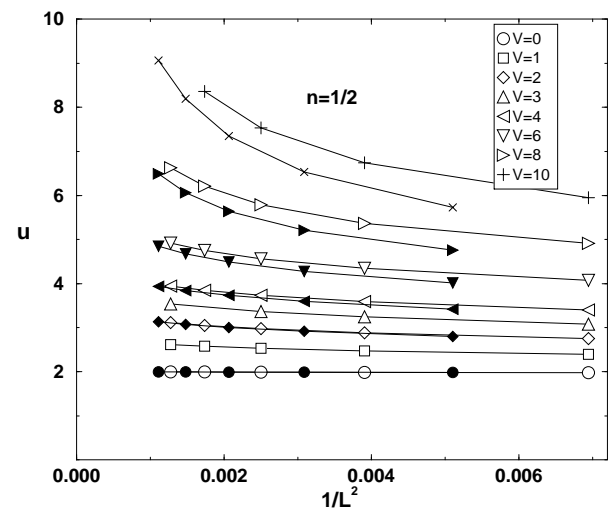

FIG. 8. Charge velocity $u$ in the long range $1 / r$ model at density $\bar{n}=1 / 2$ plotted as a function of the inverse square length. Open (closed) symbols correspond to an even (odd) number of electrons.

The anomalous exponent $K$ can be calculated for each ring size $L$ by using the conformal identity $K=\frac{D}{u}$. Such an identity can be easily derived from the quadratic form (A1) of the boson representation. This quantity is of particular importance since, in a conformal invariant theory, the charge correlations decay as a power law whose exponent is precisely given by $K$. It will also signal, as shown in the appendix, when an umklapp term becomes relevant and leads to a gap in the spectrum and an insulating behavior.

Our data for $K$ at $\bar{n}=1 / 2$ obtained for various Coulomb repulsion $V$ are plotted vs $1 / L^{2}$ in Fig. 9(a). The same set of data is shown in Fig. 9(b) as a function of $V$. For large $V, K<K_{c}$ so that umklapp scattering is expected to be strong in this regime and to produce a large charge gap in agreement with the previous findings. The results at small and intermediate $V$ are not conclusive but suggest that umklapp is weak in this regime. However, the (slow) divergence of the charge velocity should ultimately lead to $K(L) \rightarrow 0$ and then, eventually, to the 
opening of a very small charge gap at large sizes. Note that $K$ seems to be a more sensitive probe than the single particle gap itself. Indeed, the data in Fig. 9(a) for e.g. $V=4$ unambiguously show the relevance of umklapp while the extrapolation of $\Delta_{C, 1}$ in Fig. 3(b) for the same parameter is still unconclusive.
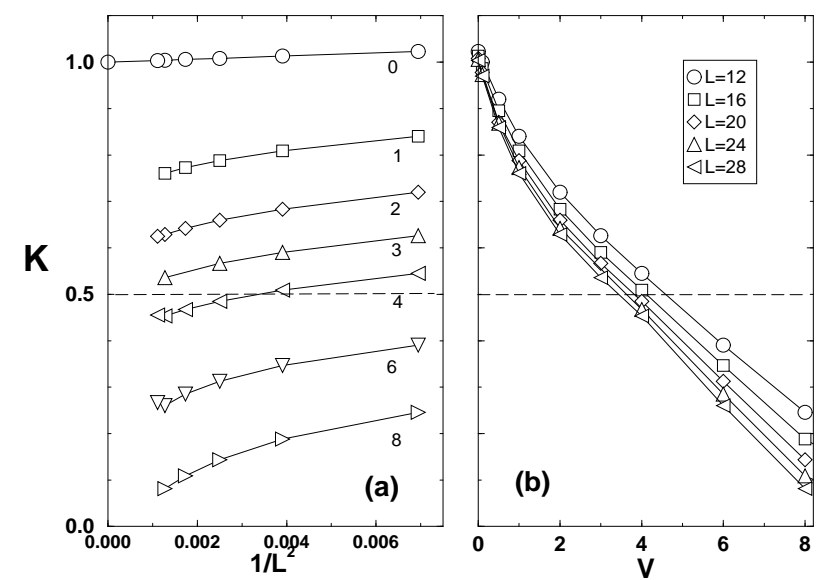

FIG. 9. Anomalous exponent $K$ (calculated from $D$ and $u$ ) of the long range $1 / r$ model at density $\bar{n}=\frac{1}{2}$. (a) Finite size behavior for different magnitudes $V$ (as shown on the plot). Note that the data obtained on the largest $L=30$ ring scale slightly differently from the data set obtained on $L=4 p$ rings; (b) vs $V$ for different sizes (as indicated in the legend). The critical value $K_{c}=1 / 2$ at half filling is shown by dotted lines on each plot.

One can get additional insights on the possible instability of the Luttinger liquid fixed point by considering a different estimation $K^{\prime}(L)$ (see e.g. 15]) of the anomalous exponent $K^{\prime}=\pi u \kappa^{-1}$, where $\kappa^{-1}$ is the inverse compressibility which can be estimated numerically as $\kappa^{-1}(L)=\frac{L}{4} \Delta_{C, 2}$ in order to avoid shell effects. In a regular LL the two estimates $K$ and $K^{\prime}$ converge toward the same thermodynamic limit within finite size corrections $\sim 1 / L^{2}$. In order to examine the breakdown of the LL character, it is then of interest to define the relative difference,

$$
\Delta K / K=\left|1-\frac{\pi u^{2}}{\kappa D}\right|
$$

This quantity is plotted vs $V$ in Figs. 10(a) and (b) for the $\mathrm{NN}$ and the long range model respectively using the same scale for a direct comparison. As expected, in the $\mathrm{NN}$ model for $V<2$ the relative ratio $\Delta K / K$ is very small (typically below 0.01) and strongly increases for $V>2$ in the insulating CDW phase. The situation is drastically different in the LR model where $\Delta K / K$ is much larger for all $V$. Note that the minimum around $V \simeq 6$ corresponds in fact to an accidental change of sign of the difference $K-K^{\prime}$ and moves continuously towards small $V$ for increasing size. We believe that Fig. 10(b) provides strong evidence that the quasi-metallic phase of the LR model is not a simple LL fixed point. It would be consistent with a system which would be a pinned (by the lattice) WC in the infinite size limit and which remains metallic of the finite size effects 11.

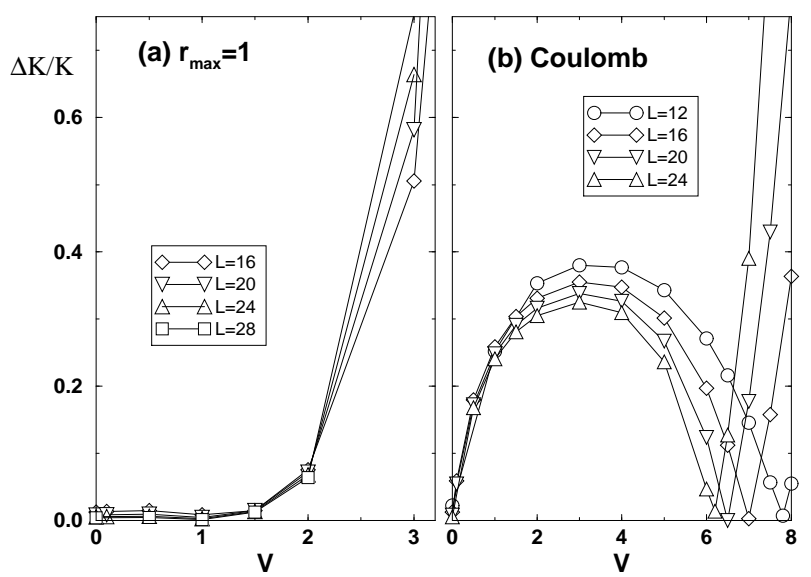

FIG. 10. Relative error $\Delta K / K$ versus $V$ at density $\bar{n}=\frac{1}{2}$ for a short range $\mathrm{NN}$ repulsion (a) and a long range Coulomb interaction (b).

\section{Low energy modes and dynamics}

Analysing the structure of the low energy spectrum for all $q$ is useful to get further understanding on the quasimetal - insulator cross-over. The dispersion of the GS vs momentum is shown in Figs. 11 at constant particle density $\bar{n}=1 / m=1 / 3$ and for two extreme values of the Coulomb repulsion, $V=2$ and $V=50$. Although one observes clearly 3 local minima at momenta 0 and $\pm 2 \pi / 3$ in both cases, the two spectra are qualitatively very different. While at small $V$ linear branches seem to appear in the vicinity of the minima, the three almost degenerate GS are clearly separated from the rest of the spectrum at large $V$ 17]. This last feature is characteristic of translation symmetry breaking occuring in a $2 k_{F}$ CDW state in which the (spinless) particles are localised at equal spacings.

A more qualitative analysis can be performed by studying the size dependence of the gap between the GS and the next excited state at momentum $q=0$ (so-called optical gap) which is shown in Fig. 12(a). At small $V$, $\Delta_{\text {opt }}$ extrapolates for $L \rightarrow \infty$ to a vanishing or small value characteristic of a metallic or quasi-metallic behavior. On the contrary, the extrapolated gap is clearly finite for large $V$ as expected in the case of an insulator. Note that, for intermediate $V$, we observe some irregular behavior of the data so that no finite size scaling analysis was attempted here. Let us point out again that the absence of gap for small $V$ is related to the finite size of the 


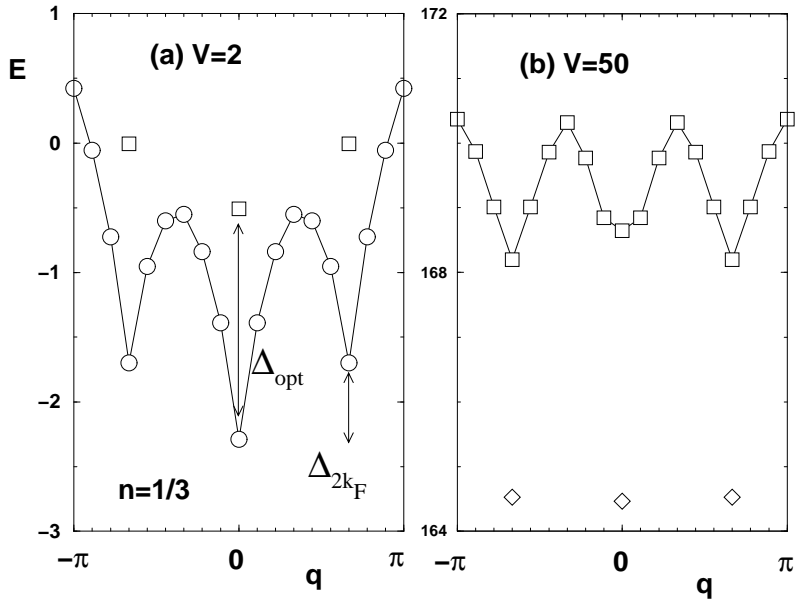

FIG. 11. Low energy spectrum of the LR Coulomb model at $\bar{n}=1 / 3$ vs momentum $q$. At momenta 0 and $2 \pi / 3$ the first excited state is also shown. The definitions of the finite size gaps $\Delta_{\text {opt }}$ and $\Delta_{2 k_{F}}$ are shown on the plot. (a) $V=2$; (b) $V=50$.

system and the fact that neither the velocity $u$ nor the Luttinger parameter $K$ have been renormalized to zero because of the log divergence of $\sum_{r} V_{r}$.

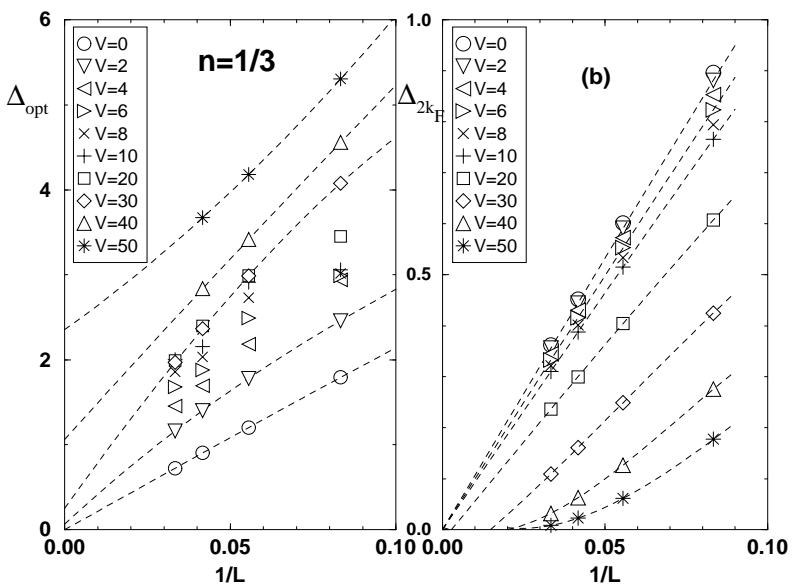

FIG. 12. Finite size scaling behaviors of the optical (a) and $2 k_{F}$ (b) gaps vs $1 / L$ for $\bar{n}=1 / 3$.

The instability towards a translation symmetry breaking CDW state can be also evidenced from the energy separation $\Delta_{2 k_{F}}$ between the absolute GS and the lowest energy at momentum $2 k_{F}$ (see Fig. 11(a) for the definition). As seen in Fig. 12(b), at small $V \Delta_{2 k_{F}}$ scales like $1 / L$, a behavior reminiscent of a LL. Expected deviations from this behavior due to logarithmic corrections can not be seen for such system sizes. For large $V, \Delta_{2 k_{F}}$ decreases faster than $1 / L$. The data for $V=40$ and $V=50$ are compatible with the expected exponential behavior for system sizes larger than the finite correlation length of the insulating CDW.

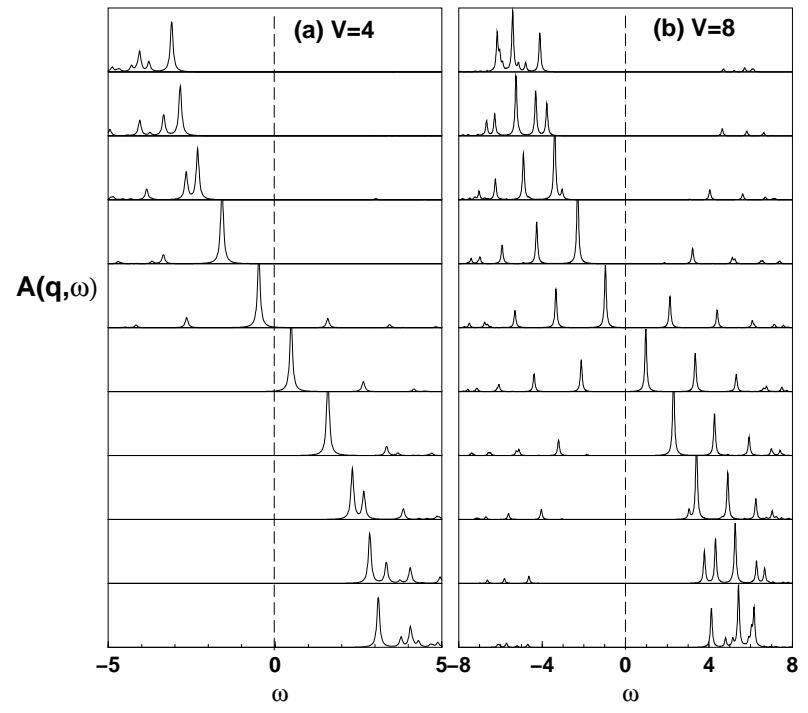

FIG. 13. Single particule spectral function vs frequency calculated on a $L=20$ sites Coulomb ring at half filling. From top to bottom, the momenta increase by equal steps from 0 to $\pi$. Energies are measured from the chemical potential. (a) $V=2 ;$ (b) $V=8$.

Lastly, we have computed the single particle spectral function $A(q, \omega)=-\frac{1}{\pi} \operatorname{Im} G(q, \omega)$, where $G(q, \omega)$ is the standard two-points Green function, and results at $\bar{n}=$ $1 / 2$ for $V=4$ and $V=8$ are shown in Figs. 13(a) and (b) respectively. For $V=4$ most of the spectral weight is located in a single dispersive branch. However, for $V=8$ when the CDW correlations become stronger, one observes clearly a quasi-symmetric "image" branch due to Bragg reflection on the CDW (fluctuating) potential. These data give a further confirmation of the cross-over occuring as the magnitude of the LR Coulomb potential increases.

\section{CONCLUSIONS}

To conclude, we have shown that, for intermediate lengthscales an interesting phenomenon occurs for a one dimensional system in the presence of long range interactions. In the infinite size limit, such a system would always be insulating due to the log divergence of the long wavelength part of the Coulomb potential $\sum_{r} V_{r}$. However, because this divergence is quite slow, for intermediate (and up to quite large !) lengthscales an opposite effect occurs due to the $2 k_{F}$ oscillations in the density: long range interactions enhance the metallic character of the system. Quite generically, umklapp scattering is reduced (but not totally suppressed) by Coulomb LR interactions. At not too large $V$ (typically $V<20$ at $n=1 / 3)$ the $1 \mathrm{D}$ electron system exhibits quasi-metallic behaviors with vanishing (or tiny) mass gaps in agree- 
ment with previous findings on spinfull LR models [11. This phase, however, shows deviations from conformal field theory predictions. A physical description in term of weakly pinned WC is proposed. At larger Coulomb interaction a cross-over towards a strongly localised $2 k_{F}$ CDW occurs. Analytical calculations based on bosonisation techniques confirm our numerical findings.

We thank IDRIS (Orsay) for allocation of CPU time on the C94 and C98 CRAY supercomputers. TG would like to thank the ITP where part of this work was completed for support (under NSF grant No. PHY94-07194) and hospitality.

Note added: While completing this work we became aware of related work by G. Fano et al. condmat $[9909140]$ in agreement with our analysis in the small $V$ regime.

[1] J. Kondo and K. Yamaji, J. Phys. Soc. Jpn. 43, 424 (1977); J. Hubbard, Phys. Rev. B 17, 424 (1978).

[2] A. Goni et al., Phys. Rev. Lett. 67, 3298 (1991).

[3] For a review see e.g. D. Jérome and H. J. Schulz, Adv. Phys. 31, 299 (1982).

[4] B. Dardel et al., Europhys. Lett. 24, 687 (1993); M. Nakamura et al., Phys. Rev. B 49, 16191 (1994). A. Sekiyama et al., Phys. Rev. B 51, 13899 (1995).

[5] H. J. Schulz, Phys. Rev. Lett. 71, 1864 (1993).

[6] L. I. Glazman and I. M. Ruzin and B. I. Shklovskii Phys. Rev. B 45, 8454 (1992).

[7] T. Giamarchi and A. J. Millis, Phys. Rev. B 46, 9325 (1992).

[8] T. Giamarchi, Physica B 230-232, 975 (1997).

[9] H. J. Schulz, "Strongly Correlated Electronic Metals", p. 187, The Los Alamos Symposium - 1993, Eds. K.S. Bedell et al., (Addison-Wesley Publishing Company, 1994).

[10] M. Mori and H. Fukuyama, J. Phys. Soc. Jpn. 65, 3604 (1996).

[11] D. Poilblanc, S. Yunoki, S. Maekawa and E. Dagotto, Phys. Rev. B 65, R1645 (1997).

[12] K. Behnia et al., Phys. Rev. Lett. 74, 5272 (1995); M. Dressel et al., ibid 77, 398 (1996).

[13] H. Frahm and V. E. Korepin, Phys. Rev. B 42, 10553 (1990); See also H. J. Schulz, "Correlated Electron Systems", p. 199, ed. V. J. Emery (World Scientific, 1993).

[14] As seen in Ref. [1], if only $V_{1}=V$ and $V_{2}=V / 2$ exist, the metallic LL phase remains stable up to $V \simeq 5.5$.

[15] H. Schulz, Phys. Rev. Lett. 64, 2831 (1990).

[16] At a density $\bar{n}=1 / q$, the classical gap is given by $2 \sum_{s=1}^{\infty} \frac{1}{(s q)^{2}-1}$; see e.g. Y. Hatsugai, Phys. Rev. B 56, 12183 (1999) and references therein.

[17] Similar behaviors have been seen for other LR models at densities $\bar{n}=1 / m$; see Ref. [16].

[18] F. D. M. Haldane, J. Phys. C 14, 2585 (1981).

[19] H. J. Schulz, in Mesoscopic quantum physics, Les Houches LXI, edited by E. Akkermans, G. Montambaux,
J. L. Pichard, and J. Zinn-Justin (Elsevier, Amsterdam, 1995).

[20] T. Giamarchi, Phys. Rev. B 44, 2905 (1991).

\section{APPENDIX A: BOSONISATION}

One can study the effect of Coulomb interations on the 1d electron gas by using a boson representation of the fermion operators (bosonisation) [18,19]. Introducing a field $\phi$ to represent the long wavelength fluctuations of the density, the kinetic energy in (11) becomes

$$
H=\frac{1}{2 \pi} \int d x u K(\pi \Pi)^{2}+\frac{u}{K}(\nabla \phi)^{2}
$$

where $\phi$ and $\Pi$ are canonically conjugate operators. For the free $(V=0)$ Hamiltonian $u=v_{F}$ and $K=1$. The parameters $u$ and $K$ are the only one necessary to describe the low energy properties of the metallic phase and are known as Luttinger liquid parameters. They can be computed in perturbation in the interaction $V$ by using the expression of the density

$$
\rho(x)=\rho_{0}-\frac{1}{\pi} \nabla \phi+\frac{1}{2 \pi \alpha} e^{i\left(2 k_{F} x+2 \phi\right)}
$$

where $\alpha$ is a short distance cutoff that can be identified with the lattice spacing. Using (A2) for the interaction term in (1) one gets

$$
\begin{aligned}
H_{\mathrm{int}} & =\sum_{i, j} V_{i-j}\left\{\frac{1}{\pi^{2}}\left(\nabla \phi_{i}\right)\left(\nabla \phi_{j}\right)\right. \\
& \left.+\frac{1}{(2 \pi \alpha)^{2}} 2 \cos \left(2 k_{F}\left(x_{i}-x_{j}\right)+2 \phi_{i}-2 \phi_{j}\right)\right\} .
\end{aligned}
$$

By considering that the fields $\phi$ are slowly varying at the scale of the lattice one can expand (A3). To expand safely the fields should be normal ordered using

$$
e^{i 2\left(\phi\left(r_{1}\right)-\phi\left(r_{2}\right)\right)}=: e^{i 2\left(\phi\left(r_{1}\right)-\phi\left(r_{2}\right)\right.}: e^{-\frac{1}{2}\left\langle\left[2\left(\phi\left(r_{1}\right)-\phi\left(r_{2}\right)\right)\right]^{2}\right\rangle} .
$$

At the lowest order in $V$, (A4) becomes

$$
e^{i 2\left(\phi\left(r_{1}\right)-\phi\left(r_{2}\right)\right)}=: e^{i 2\left(\phi\left(r_{1}\right)-\phi\left(r_{2}\right)\right.}: \frac{\alpha^{2}}{\left(r_{1}-r_{2}\right)^{2}}
$$

In the normal product one can expand $\phi\left(r_{1}\right)-\phi\left(r_{2}\right) \simeq$ $\left(r_{1}-r_{2}\right) \nabla \phi(R)$ where $R=\left(r_{1}+r_{2}\right) / 2$. One thus recovers a quadratic form similar to A1 but with

$$
\begin{aligned}
u / K & =v_{F}+\frac{2}{\pi} \sum_{r} V_{r}\left(1-\cos \left(2 k_{F} r\right)\right) \\
u K & =v_{F}
\end{aligned}
$$

where $r=1,2,3, \ldots$ is the distance on the lattice. The above expression is valid at the lowest order in $V$. For 
finite $V$ the various coefficients in it will get renormalized by irrelevant operators. For a long range potential the $q \rightarrow 0$ part is dominant and gives rise to the logarithmic divergence of $A_{q}$ responsible for the one dimensional plasmon. Due to the $2 k_{F}$ oscillations in density the $2 k_{F}$ component of the potential also occurs. Note that although (A6) looks superficially similar to what one could obtain using g-ology arguments, such arguments would naively suggest that $g_{1}=-g_{2}$. This would lead the $V\left(2 k_{F}\right)$ part to enter both the $u / K$ and $u K$ term and lead to an incorrect renormalization of the $u K$ term. Such a change of $u K$ is impossible for a well defined lattive model [20], where one should have $u K=v_{F}$ as a consequence of Galilean invariance in the absence of an underlying lattice.

In the presence of a lattice one should take into account that the momentum is only conserved modulo a vector of the reciprocal lattice (i.e. for example for half filling $\left.4 k_{F}=2 \pi\right)$. When taking this effect into account (A3) gives also rise to the so-called umklapp terms. These terms are the one responsible for the Mott transition and the opening of the charge gap [8]. They can also be computed, and read e.g. for the half filled case

$$
H_{\mathrm{umk}}=\frac{-2 g}{(2 \pi \alpha)^{2}} \int d x \cos (4 \phi(x))
$$

where

$$
g=\sum_{r} V_{r} \cos \left(2 k_{F} r\right) .
$$

Taken together with (A1), (A7) is able to open a gap in the spectrum and lead to a Mott insulator (or equivalently to a Wigner crystal pinned on the lattice) for $K<K_{c}$, where $K_{c}$ depends on the commensurability. For half filling $K_{c}=1 / 2$ and would be $K_{c}=1 /\left(2 n^{2}\right)$ for a commensurability of order $n$. 